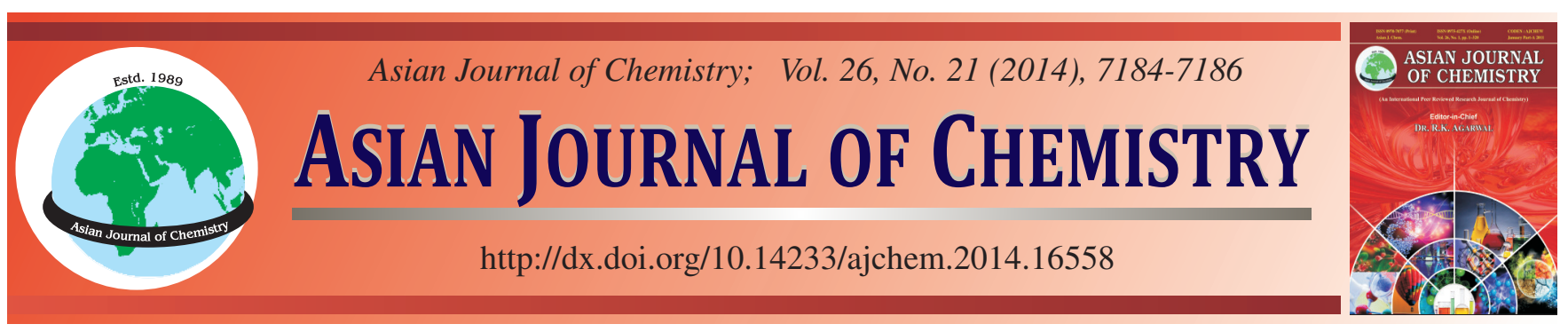

\title{
Effect of Flux $\mathrm{NH}_{4} \mathrm{~F}$ on Structure and Luminescence Properties of $\mathrm{SrMoO}_{4}: \mathrm{Tb}^{3+}$ Phosphors Synthesized by Microwave Radiation Method
}

\author{
Yong-Qing Zhai ${ }^{1}$, , Rui-Fang Li ${ }^{1}$, Yao-Xuan CuI ${ }^{1}$, Shan-Shan Song ${ }^{1}$ and Jia Qiao ${ }^{2}$
}

${ }^{1}$ College of Chemistry and Environmental Science, Hebei University, Baoding 071002, P.R. China

${ }^{2}$ College of Mechanical and Electrical Engineering, Agricultural University of Hebei, Baoding 071002, P.R. China

*Corresponding author: E-mial: zhaiyongqinghbu@163.com

Received: 21 October 2013;

Accepted: 21 January 2014;

Published online: 30 September 2014;

AJC-16098

\begin{abstract}
$\mathrm{SrMoO}_{4}: \mathrm{Tb}^{3+}$ green-emitting phosphors were prepared by microwave radiation method at medium-high power ( $560 \mathrm{~W}$ ) for $0.5 \mathrm{~h}$ using active carbon as microwave absorbent and $\mathrm{NH}_{4} \mathrm{~F}$ as the flux. The effect of flux $\mathrm{NH}_{4} \mathrm{~F}$ on crystal structure, morphology and luminescent properties of samples were investigated by X-ray powder diffraction, scanning electron microscope and fluorescence spectrophotometer respectively. The results show that $\mathrm{NH}_{4} \mathrm{~F}$-added samples are Scheelite-type tetragonal phase $\mathrm{SrMoO}_{4}$ with space group $\mathrm{I}_{1 / \mathrm{a}}$. The particles of $\mathrm{SrMoO}_{4}$ : $\mathrm{Tb}^{3+}$ phosphors prepared with flux $\mathrm{NH}_{4} \mathrm{~F}$ have smooth surface and are larger than that of sample with no flux. With the increase of $\mathrm{NH}_{4} \mathrm{~F}$ dosage, the location and shape of the excitation and emission spectra have no obvious change, but the luminescent intensity strongly depends on the dosage of $\mathrm{NH}_{4} \mathrm{~F}$. When the dosage of $\mathrm{NH}_{4} \mathrm{~F}$ is $4 \mathrm{wt} \%$, the emission intensity reaches to the maximum. Compared with the sample without flux, the intensity is enhanced about $58 \%$.
\end{abstract}

Keywords: Microwave radiation method, $\mathrm{SrMoO}_{4}: \mathrm{Tb}^{3+}$, Green phosphors, Flux, $\mathrm{NH}_{4} \mathrm{~F}$, Luminescence.

\section{INTRODUCTION}

Recently, white light emitting diodes (WLEDs) with small size, high luminous reliability, long lifetime, low energy consumption and environment-friendly characteristics ${ }^{1,2}$ have attracted much attention due to its widespread technological applications, such as backlighting for liquid crystal displays, incandescent and auto lamps, the landscape lighting and other emerging areas $^{3-6}$.

It is well known that there are two approaches to generating white light in light emitting diodes. One is to mix different color lights emitted directly by tricolor chips; the other is to mix different colors obtained by converting the emission of blue or ultraviolet LED chip to longer-wavelength light using phosphors $^{4,7}$. Considered the feasibility, practicability, commercialization and other aspects, the latter is the main development direction of WLEDs in the future. Therefore, to develop tricolor phosphors with high conversion efficiency under UV excitation has important significance and has become research emphasis in solid-state lighting at present. On one hand, green-emitting phosphors can be used for the preparation of pure green LEDs and on the other hand, it can be used to form the green part in the three primary colors of WLEDs ${ }^{8}$.

Luminescence properties of phosphors strongly depend on the crystallization degree, particle size and morphology. Usually, the fluxes have a great influence on the ionic diffusions, crystallization and the formation of the target in high temperature solid reaction ${ }^{9}$. Proper fluxing agent is added into the system to decrease melting point and improve the diffusion rate and the rate of solid reaction. For luminescent materials, it is beneficial to the formation of the matrix, the doped ions entering into matrix lattice easily and improving luminescence intensity of product ${ }^{10,11}$.

In the present work, green-emitting phosphors $\mathrm{SrMoO}_{4}: \mathrm{Tb}^{3+}$ were prepared by microwave radiation method. This method has a lot of advantages, such as fast and efficient, simple operation and good heating uniformity. It provides a new idea for saving energy and reducing energy consumption. The influences of fluxing agent $\mathrm{NH}_{4} \mathrm{~F}$ on the crystal structure, morphology and luminescent properties of the obtained samples were investigated. The obtained samples exhibits excellent luminescent properties and can be excited effectively by UV at about $287 \mathrm{~nm}$ and show strong green emitting. Therefore, as-synthesized $\mathrm{SrMoO}_{4}: \mathrm{Tb}^{3+}$ shows good prospect for green phosphors of WLEDs.

\section{EXPERIMENTAL}

The powder samples of $\mathrm{SrMoO}_{4}: \mathrm{Tb}^{3+}$ were prepared by microwave radiation method. The starting materials were $\mathrm{Tb}_{4} \mathrm{O}_{7}, \mathrm{MoO}_{3}$ and $\mathrm{SrCO}_{3}$, with purity of $99.9,99.9999$ and $99.9 \%$, respectively. The other materials used in this study 
include $\mathrm{NH}_{4} \mathrm{~F}$ (A.R.) as flux and active carbon (A.R.) as microwave absorbent.

The preparation process of $\mathrm{SrMoO}_{4}: \mathrm{Tb}^{3+}$ is as follows. According to the stoichiometric composition of $\mathrm{SrMoO}_{4}: \mathrm{Tb}^{3+}$, starting materials $\mathrm{Tb}_{4} \mathrm{O}_{7}, \mathrm{MoO}_{3}$ and $\mathrm{SrCO}_{3}$ were mixed together with a certain amount of $\mathrm{NH}_{4} \mathrm{~F}$ and ground in a porcelain mortar for $0.5 \mathrm{~h}$ to ensure homogeneity and fine particle size. The mixture was placed in a corundum crucible inside a large covered ceramic crucible. The space between the corundum and the ceramic crucible was filled with active carbon as microwave absorbent or heating medium. Then, the crucibles were placed into a Galanz WG700SL2011 -KG microwave oven and heated for $0.5 \mathrm{~h}$ under the middle-high power $(560 \mathrm{~W})$. Subsequently, the samples were cooled to room temperature in air and ground into powder in a porcelain mortar to obtain the desired products.

Characterization: The crystal structure and the phase purity of the samples were examined by X-ray powder diffraction (XRD) using $\mathrm{CuK}_{\alpha}$ radiation $(30 \mathrm{kV} \times 20 \mathrm{~mA}$ and a scanning speed $0.06 \%$ s). The particle size and morphology of the samples were characterized with a scanning electron microscopy (SEM). The excitation and emission spectra of the samples were recorded on an F-380 fluorescence spectrophotometer. All measurements were carried out at room temperature.

\section{RESULTS AND DISCUSSION}

In order to investigate the influence of flux $\mathrm{NH}_{4} \mathrm{~F}$ dosage on the crystal structure and phase purity of samples, a series of samples have been prepared with different dosage of $\mathrm{NH}_{4} \mathrm{~F}$ varying between 0 and $10 \mathrm{wt} \%$. The XRD patterns of the assynthesized samples are shown in Fig. 1. It can be seen that the sample synthesized without flux exhibits $\mathrm{SrMoO}_{4}$ phase together with a little amount of raw materials $\mathrm{MoO}_{3}$, which indicates that the reaction proceeded incompletely. When $\mathrm{NH}_{4} \mathrm{~F}$ is introduced as the flux, the peaks of $\mathrm{MoO}_{3}$ phase become weak. When the dosage of $\mathrm{NH}_{4} \mathrm{~F}$ is increased to $4 \mathrm{wt} \%$, the $\mathrm{MoO}_{3}$ phase disappears and the XRD pattern is in good agreement with JCPDS card (No. 85-0809). So, the sample is pure Scheelite-type tetragonal structure $\mathrm{SrMoO}_{4}$ with space group

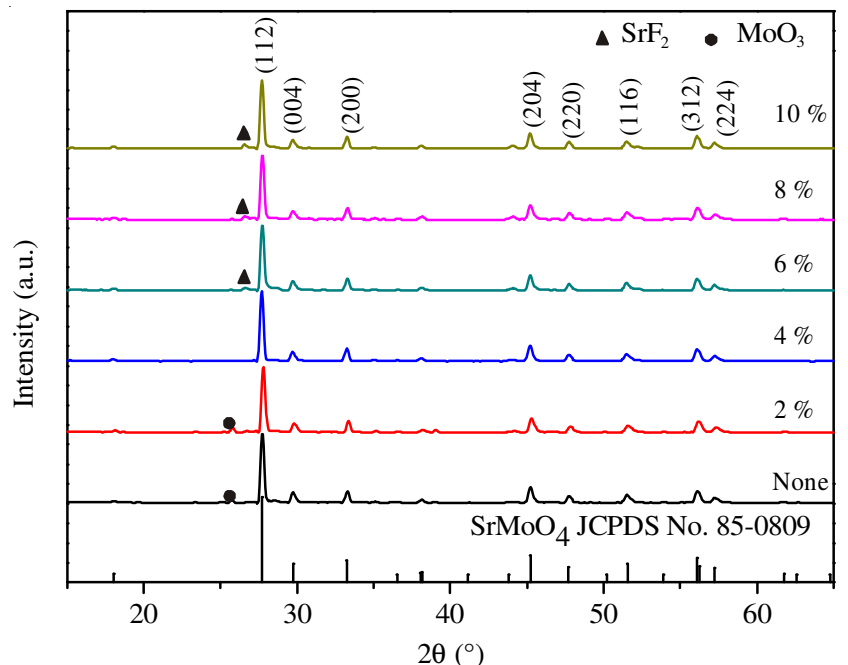

Fig. 1. XRD patterns of samples obtained under different dosage of flux $\mathrm{NH}_{4} \mathrm{~F}$
I $4_{1 / a}$. No peaks of $\mathrm{Tb}$ compounds can be observed, which suggests that $\mathrm{Tb}^{3+}$ ions have been uniformly incorporated into the host lattice of $\mathrm{SrMoO}_{4}$. The sharp and narrow XRD peaks indicate that as-synthesized $\mathrm{SrMoO}_{4}: \mathrm{Tb}^{3+}$ phosphors have high crystallinity. When the amount of $\mathrm{NH}_{4} \mathrm{~F}$ is over $4 \mathrm{wt} \%$, new phase $\mathrm{SrF}_{2}$ appears and the intensity of diffraction peaks of $\mathrm{SrF}_{2}$ increases slightly with the increase of $\mathrm{NH}_{4} \mathrm{~F}$ dosage.

SEM analysis: The effects of flux $\mathrm{NH}_{4} \mathrm{~F}$ on the morphology and particle size of $\mathrm{SrMoO}_{4}: \mathrm{Tb}^{3+}$ phosphors have been studied. Fig. 2 illustrates SEM images of phosphors prepared with no flux and $4 \mathrm{wt} \% \mathrm{NH}_{4} \mathrm{~F}$. It can be seen that the particles of $\mathrm{SrMoO}_{4}: \mathrm{Tb}^{3+}$ phosphors prepared with the addition of $\mathrm{NH}_{4} \mathrm{~F}$ have larger size and clearer interface compared with the sample without flux, which suggests that the introduction of flux $\mathrm{NH}_{4} \mathrm{~F}$ promotes the growth of $\mathrm{SrMoO}_{4}: \mathrm{Tb}^{3+}$ particles, enhance the crystallization degree and decrease surface defect.
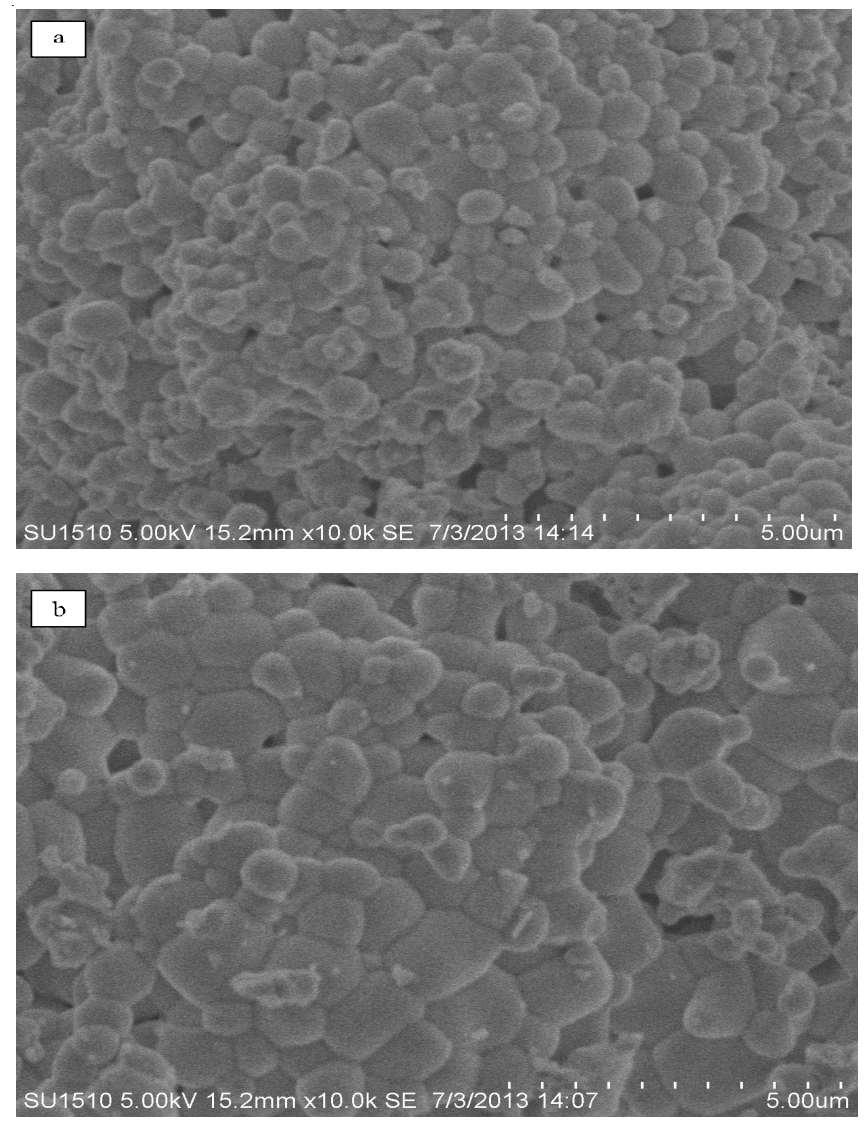

Fig. 2. SEM images of $\mathrm{SrMoO}_{4}: \mathrm{Tb}^{3+}$ phosphor synthesized with different dosage of $\mathrm{NH}_{4} \mathrm{~F}$, (a) 0 wt \%, (b) 4 wt \%

Luminescent properties: In order to investigate the influence of flux $\mathrm{NH}_{4} \mathrm{~F}$ dosage on the photoluminescence properties of the $\mathrm{SrMoO}_{4}: \mathrm{Tb}^{3+}$ powders, the as-synthesized samples were characterized by the photoluminescence excitation and emission spectra.

Excited by $287 \mathrm{~nm}$, the emission spectra of samples were measured and shown in Fig. 3. It can be seen that the dosage of $\mathrm{NH}_{4} \mathrm{~F}$ has little effect on the shape and position of emission spectra. All the emission spectra are composed of a group of narrow peaks at about 490, 544, 586 and $621 \mathrm{~nm}$, which can be ascribed to ${ }^{5} \mathrm{D}_{4}{ }^{7} \mathrm{~F}_{\mathrm{J}}(J=6,5,4,3)$ electronic transition of $\mathrm{Tb}^{3+}$, respectively ${ }^{9}$. The main emission peak at $544 \mathrm{~nm}$ due to 


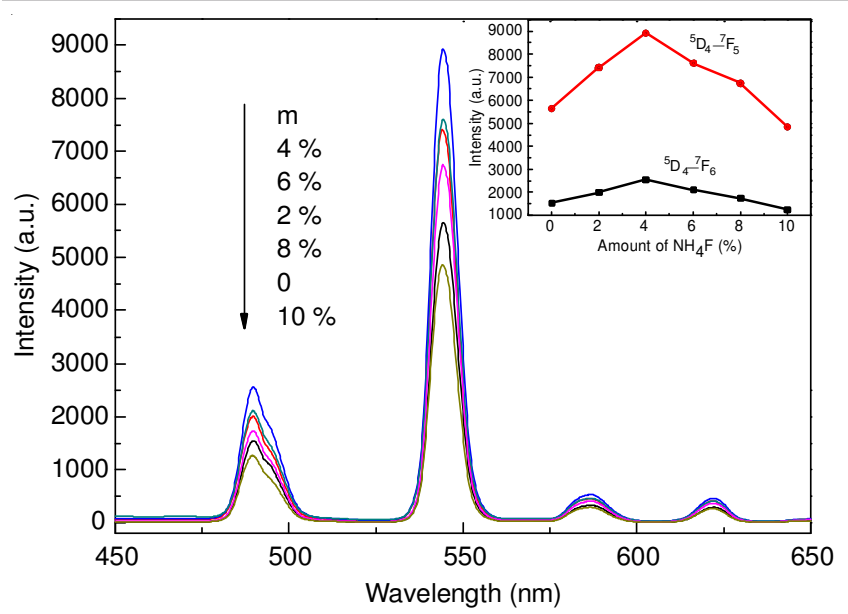

Fig. 3. Emission spectra of $\mathrm{SrMoO}_{4}: \mathrm{Tb}^{3+}$ with different dosage of $\mathrm{NH}_{4} \mathrm{~F}$

${ }^{5} \mathrm{D}_{4-}{ }^{7} F_{5}$ transition of $\mathrm{Tb}^{3+}$. It is worth noting that the dosage of $\mathrm{NH}_{4} \mathrm{~F}$ has great effect on the luminescent intensity. The intensity of emission peak at $544 \mathrm{~nm}$ first increases with the increase of $\mathrm{NH}_{4} \mathrm{~F}$ dosage, then decreases when $\mathrm{NH}_{4} \mathrm{~F}$ dosage exceeds 4 wt \%(as shown in the inset in Fig. 3). The intensity of emission peak at $544 \mathrm{~nm}$ of as-prepared $\mathrm{SrMoO}_{4}: \mathrm{Tb}^{3+}$ phosphor with $4 \mathrm{wt} \% \mathrm{NH}_{4} \mathrm{~F}$ reaches the maximum, which is 1.58 times of the sample with no flux. Therefore, it can be known that the appropriate doping amount of flux $\mathrm{NH}_{4} \mathrm{~F}$ can improve luminescent intensity of the sample significantly.

Fig. 4 shows the excitation spectra of $\mathrm{SrMoO}_{4}: \mathrm{Tb}^{3+}$ samples, which was obtained by monitoring the ${ }^{5} D_{4^{-}}{ }^{7} F_{5}$ transition emission of $\mathrm{Tb}^{3+}$ at $544 \mathrm{~nm}$. It can be seen that the shape of excitation spectra is similar and all of them consist of a broad band between 200 and $350 \mathrm{~nm}$ with a main peak at about $287 \mathrm{~nm}$ and some weak peaks between $350 \mathrm{~nm}$ and $500 \mathrm{~nm}$. The broad band is ascribed to the charge-transfer band of Mo-O and $4 f^{8}-4 f^{7} 5 d^{1}$ transition of $\mathrm{Tb}^{3+}$. The weak peaks belong to $4 f-4 f$ transition of $\mathrm{Tb}^{3+12}$. The dosage of $\mathrm{NH}_{4} \mathrm{~F}$ has little effect on the shap and position of the excitation spectra. But,the peak intensity of excitation spectra strongly depends on the dosage of $\mathrm{NH}_{4} \mathrm{~F}$, it has the same trend as that of the emission spectra along with the dosage of $\mathrm{NH}_{4} \mathrm{~F}$ (as shown in the inset in Fig. 4).

The possible reason for above results is as follows. Luminescence properties have a lot to do with the phase purity and crystallinity of the sample. The more pure, more complete crystal phase is, the better luminescence properties perform. By the addition of flux agent at low concentration level in a phosphor material, the crystallinity of host lattice is improved by assisting ionic diffusion during high temperature processing and when the flux dosage is over a certain range, the excessive amount of liquid phase has an impact on the crystallization, destroy the luminescence center and the host structure to some extent and make the luminescent performance become poor, because of liquid viscosity increasing, the deposition of extra flux, crystal reunion and agglomeration between the particles in the process of cooling ${ }^{13}$. When $\mathrm{NH}_{4} \mathrm{~F}$ dosage exceeds $4 \mathrm{wt}$ $\%$, the generation of new phase $\mathrm{SrF}_{2}$ (Fig. 1) as impurity makes the luminescence efficiency of $\mathrm{Tb}^{3+}$ decrease, at the same time, transparent vitreous material was observed in the process of experiment. Comprehensive consideration of the crystal

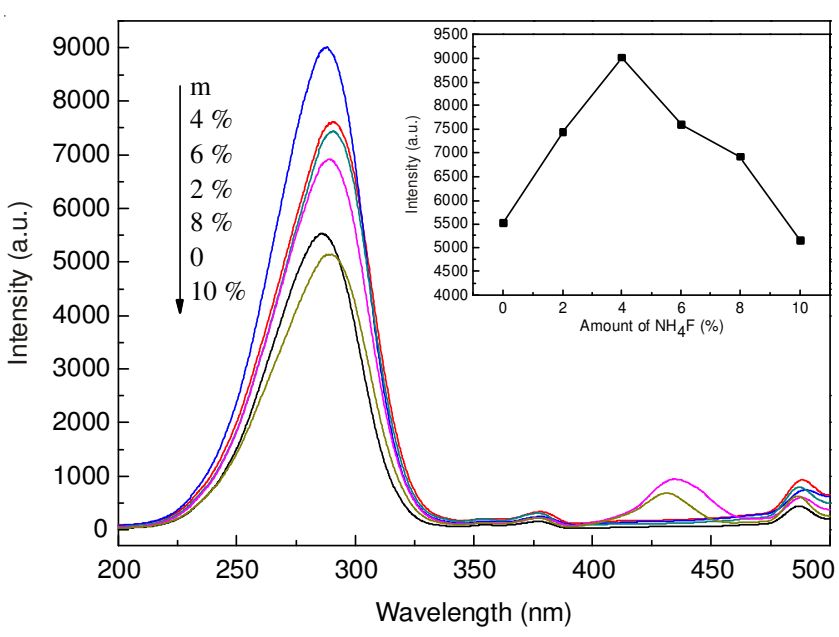

Fig. 4. Excitation spectra of $\mathrm{SrMoO}_{4}: \mathrm{Tb}^{3+}$ with different dosage of $\mathrm{NH}_{4} \mathrm{~F}$

structure, phase purity and luminescence properties, the optimum dosage of $\mathrm{NH}_{4} \mathrm{~F}$ is 4 wt \%.

\section{Conclusion}

In the present work, green phosphors $\mathrm{SrMoO}_{4}: \mathrm{Tb}^{3+}$ has been synthesized by microwave radiation method, in which $\mathrm{NH}_{4} \mathrm{~F}$ were used as the flux. The results show that the introduction of flux $\mathrm{NH}_{4} \mathrm{~F}$ is conductive to the improvement of phase purity and crystallinity of $\mathrm{SrMoO}_{4}: \mathrm{Tb}^{3+}$. Meanwhile, flux $\mathrm{NH}_{4} \mathrm{~F}$ is helpful to increase the particle size and decrease surface defect. The luminescent intensity of $\mathrm{SrMoO}_{4}: \mathrm{Tb}^{3+}$ can be enhanced obviously by adding proper amount of $\mathrm{NH}_{4} \mathrm{~F}$ and reaches the maximum when the dosage of $\mathrm{NH}_{4} \mathrm{~F}$ is 4 wt \%. Compared with the sample with no flux, the intensity is enhanced about $58 \%$. Therefore, the obtained $\mathrm{SrMoO}_{4}: \mathrm{Tb}^{3+}$ powders exhibit good prospect for green phosphors of white light emitting diodes because of their excellent luminescence performance.

\section{ACKNOWLEDGEMENTS}

This study was supported by National Natural Science Foundation of China (No. 20675023).

\section{REFERENCES}

1. Y.Q. Zhai, Z.J. You, Y.H. Liu, Y.P. Sun and Q.Q. Ji, J. Rare Earths, 30, 114 (2012)

2. A. Xie, X.M. Yuan, F.X. Wang, Y. Shi, J. Li, L. Liu and Z.F. Mu, J. Alloys Comp., 501, 124 (2010).

3. X.H. He, J. Zhou, N. Lian, J.H. Sun and M.Y. Guan, J. Lumin., 130, 743 (2010)

4. Z.L. Wang, H.B. Liang, M.L. Gong and Q. Su, J. Alloys Comp., 432, 308 (2007)

5. Md. Haque, H. I. Lee and D. K. Kim, J. Alloys Comp., 481, 792 (2009).

6. P.L. Li, Z.J. Wang, Z.P. Yang, Q.L. Guo and X. Li, J. Lumin., 130, 22 (2010).

7. X. Li, Z.P. Yang, L. Guan and Q.L. Guo, Mater. Lett., 63, 1096 (2009).

8. S.X. Cao, T. Han and M.J. Tu, Mater. Rev., 25, 65 (2011).

9. N. Niu, P.P. Yang, W.X. Wang, F. He, S.L. Gai, D. Wang and J. Lin, Mater. Res. Bull., 46, 333 (2011).

10. D.M. Yang, H.X. Ji, L.M. Xie, L. Wei and W.Y. Hu, J. Chin. Ceram. Soc., 39, 1239 (2011).

11. S.Q. Xu, L.Z. Sun, Y. Zhang, H.D. Ju, S.L. Zhao, D.G. Deng, H.P. Wang and B.L. Wang, J. Rare Earths, 27, 327 (2009).

12. A.P.A. Marques, F.V. Motta, M.A. Cruz, J.A. Varela, E. Longo and I.L.V. Rosa, Solid State Ion., 202, 54 (2011).

13. Z.Y. Feng, W.D. Zhuang, X.W. Huang, X.F. Wen and Y.S. Hu, J. Rare Earths, 28, 351 (2010). 